\title{
Development of associative and perceptual interference
}

\author{
G. ROBERT GRICE, LYN CANHAM, and CHARLES SCHAFER \\ University of New Mexico, Albuquerque, New Mexico
}

\begin{abstract}
In a choice reaction-time letter-identification task, the temporal development of perceptual and associative interference was studied with visual displays containing identical, irrelevant, or response-incompatible noise letters. Using the methods of variable-criterion theory, it was determined that perceptual interference is complete very early and has no effect on the shape of the function for the growth of associative strength. Associative interference begins later, gradually increases to a maximum, and then declines to zero at long latencies. In experiments with speeded performance, it was also found that the growth of excitatory strength for errors contains the same nonmonotonic process as for correct responses, but in reverse form. In addition, it was observed that the discriminability of the target stimuli affects the rate of growth of associative strength for the correct response. Analyses of individual differences in theoretical parameters indicate information-processing patterns similar to those reported for simple auditory tasks.
\end{abstract}

Since the original presentation of variable-criterion theory by Grice (1968), this approach and its methods of analysis have been directed toward the investigation of variables in human conditioning and reaction time (RT). The major aim has been the development of quantitative theory of increasing generality. Recently, the theory has been developed to the point at which it is able to provide a unified account of the three classical forms of RT experiments described by Donders (Grice, Nullmeyer, \& Spiker, 1982). To date, however, the research has been limited to situations in which the response is to simple stimuli such as lights and tones. Here, the approach was directed toward the analysis of recent problems that have arisen in the area of letter identification.

In the context of RT experiments of letter identification, C. W. Eriksen and Schultz (1979) have presented what they term a "continuous flow conception." According to this view, information about the stimulus increases gradually and continuously. But, while stimulus information continues to grow, responses are continuously partially activated or primed. When response priming reaches an evocation threshold, the response occurs. Although in different language, this conception is equivalent to the concept of associative strength used in variable-criterion theory. Grice (1972) suggested that associative strength increases as a function of time elapsed since stimulus onset, and presented a quantitative function

This research was supported by PHS Grant MH 16400 from the National Institute of Mental Health. Requests for reprints should be sent to G. Robert Grice, Department of Psychology, University of New Mexico, Albuquerque, New Mexico 87131. describing its growth. Since then, the conception has continued to characterize the approaches to RT and conditioning based on variable-criterion theory. The probabilistic property of response latency is based upon a normally distributed criterion or threshold. Other features of the C. W. Eriksen and Schultz (1979) conception are that the specificity of priming to the correct response increases with time and with the distinctiveness of stimuli associated with competing responses. These features also characterize variable-criterion theory and, in the more recent versions, are related to the concept of associative discrimination, which holds that associative discrimination grows in the time after the stimulus has been presented (Grice, Spiker, \& Nullmeyer, 1979).

The research reported by C. W. Eriksen and Schultz (1979) involved choice reaction-time (CRT) tasks of letter identification in which the visual display consisted of the target letter flanked on each side by a noise letter. In this situation, subjects are unable to avoid processing the noise letter even though instructed to ignore it. One finding of special interest was that if the target and noise letters were response incompatible, that is, assigned to competing responses, $\mathrm{RT}$ was longer than if they were response compatible or if the noise letter was irrelevant. This fact was first reported by B. A. Eriksen and C. W. Eriksen (1974). We believe this to be an important discovery for at least two reasons. First, it demonstrates an important role of the response and associative processes in a situation previously regarded as dependent on perceptual factors only. Second, it provides a new and potentially powerful technique for investigating the dynamics of associative inter- 
ference. The present research attempts to capitalize on the second of these reasons.

As with most other current information-processing research using $\mathrm{RT}$ measures, the work related to the continuous-flow conception has relied exclusively on comparisons of mean RT. The methods of variablecriterion theory, on the other hand, make it possible to examine functions describing the growth of processes leading to response evocation in the time following stimulus presentation. This adds a considerable amount of information concerning the dynamics of these processes and may lead to further hypotheses concerning their nature. Here, we propose to examine the temporal development of associative interference and to compare it with that of interference of purely perceptual origin.

The experiments reported here are CRT experiments in which the target letter is flanked on each side by a single letter. In each experiment there were three noise conditions: noise letter identical to the target; noise letter irrelevant to the task; and responseincompatible noise, in which the noise letter was that assigned to the competing response. The difference between the RTs for identical and irrelevant noise is considered to indicate the effect of perceptual interference, and an additional effect of incompatible noise is referred to as associative interference. Presumably, the incompatible-noise condition contains both sources of interference. We used the identical-noise condition rather than a no-noise condition because we wished all displays to be of approximately equal total energy. However, C. W. Eriksen and Schultz (1979) found no noise and identical noise to be about the same. ${ }^{1}$ Three of these experiments were conducted under accuracy instructions; two were conducted under speeded conditions in order to obtain the additional information to be gained from higher error rates.

\section{METHOD}

\section{Subjects}

The subjects were 140 women who were students in courses in introductory psychology. They received course credit for their participation. There were 28 subjects in each of the five experiments.

\footnotetext{
Apparatus and Stimuli

The experiment was controlled, the stimuli presented, and the RTs recorded by means of a TRS-80 microcomputer in combination with LVB Corp. interface devices. The subject was seated in a sound-attenuating chamber at a small table in front of a remote TV monitor. On the table were two telegraph keys operated by the subject's two index fingers. The stimuli were the regular TRS-80 double-width ( 32 characters per line) uppercase letters, which are formed by a $5 \times 7$ dot matrix. They were light against a dark background. The luminance of a dark portion of the screen was $.74 \mathrm{~cd} / \mathrm{m}^{2}$, and that of a fully lighted portion of the screen was $8.49 \mathrm{~cd} / \mathrm{m}^{2}$. Measurements were with a Spectra brightness spot meter. The symbol + appeared in the middle of the screen as a fixation point between trials and went off as the
}

stimuli came on. Visual angles reported here are approximate, since no head restraint was used. However, positioning of the table and keys did not allow much variation. The fixation point was $.38 \mathrm{deg}$ high and $.43 \mathrm{deg}$ wide. The letters were $.53 \mathrm{deg}$ high and $.43 \mathrm{deg}$ wide. The target letter appeared $.45 \mathrm{deg}$ directly above the fixation point. The noise letter was replicated on both sides of the target at a distance of $.5 \mathrm{deg}$. The nominal viewing distance to the screen was $76 \mathrm{~cm}$. RTs were recorded in microseconds. Procedures by means of which we achieved 1-msec accuracy with respect to stimulus presentation and timing have been described elsewhere by Grice (1981).

\section{Procedure}

The subjects were instructed to respond to one of the target letters with the right key and to the other with the left. The rightleft relation was reversed for half of the subjects in each experiment. Since this produced no significant effects, it is ignored in all analyses presented here. The subjects were also told to attend to the middle letter only and to ignore the others. A warning signal before each trial consisted of a $.5-\mathrm{sec} 1,000-\mathrm{Hz}$ tone of $70 \mathrm{~dB}$ SPL presented through earphones. The interval from the onset of the warning signal to stimulus onset was $1.0,1.2,1.5$, 1.8 , or $2.0 \mathrm{sec}$ equally often and in an irregular order. Stimuli remained on until the subject responded. The experiment began with 50 practice trials. These were followed by a short rest, during which any questions were answered and any necessary reinstruction given. This was then followed by 250 trials without interruption. The first 10 of these were treated as further practice; the data were based on the final 240 trials, 80 with each kind of noise. The three noise types were also equated in practice and, throughout, were administered in an irregular order. The interval between trials was $4 \mathrm{sec}$. Any trial on which a response had not occurred by $1,600 \mathrm{msec}$ was terminated. These trials and others over $1,500 \mathrm{msec}$ were not included in computing subject statistics. Such trials were rare, being less than $1 \%$.

Accuracy experiments. Three experiments were conducted with instructions to respond as quickly as possible to the target letter while avoiding errors. In all of the experiments, the three letters $A, H$, and $K$ were used as stimuli. We shall designate the experiments according to the following system: the first two letters indicate the target stimuli and the third indicates the letter used in the irrelevant-noise condition. Thus, the three experiments were AHK, AKH, and HKA.

When an error occurred in these experiments, the word "ERROR" appeared on the screen for .5 sec and a .5-sec burst of noise ( $70 \mathrm{~dB}$ SPL) sounded in the earphones.

Deadline experiments. Experiments AHK and HKA were replicated under a deadline procedure in order to induce speeding and thus a higher error rate. A separate deadline was used for each subject. The first 50 practice trials were conducted under the accuracy instructions. The final 30 of these trials consisted of 10 of each of the three noise conditions, and the mean of these 30 trials was computed. This mean became the deadline for the rest of the experiment. During the break, the subject was reinstructed that the deadline now existed and that she should attempt to meet it on every trial. When a subject failed to meet the deadline, the word "SLOW" appeared on the screen and the burst of noise sounded in the earphone. For errors, the word "ERROR" appeared, but the noise was not used.

\section{RESULTS}

Means of subject mean RTs for correct responses for the three conditions in each experiment are presented in Table 1. Results of the statistical comparisons made are presented in Table 2 . The appropriate comparison to evaluate the effect of perceptual interference is between identical and irrelevant noise. 
Table 1

Means and Standard Deviations of Mean Reaction Time (in Milliseconds) and of Error Rate for Each Noise Condition in Each Experiment

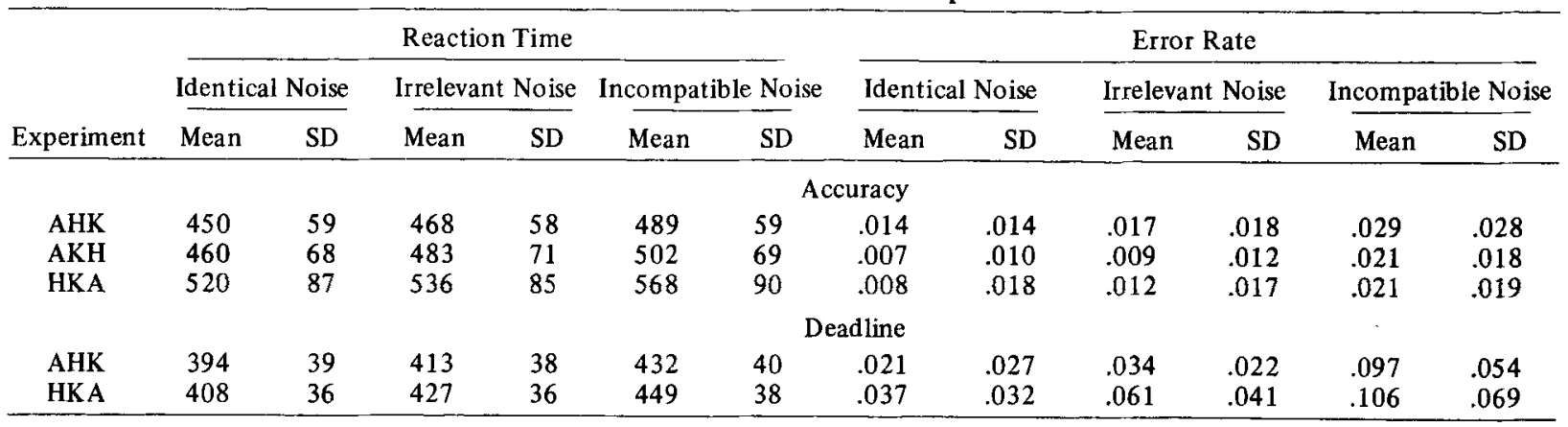

Since the incompatible-noise condition also involves perceptual interference, the appropriate comparison for associative interference is between irrelevant and incompatible noise. These comparisons have been made in combination with comparisons of pairs of experiments. Comparison of the accuracy experiments showed that AHK and AKH did not differ significantly, but that experiment HKA was significantly slower than both. This suggests that HK was a more difficult discrimination than $\mathrm{AH}$ or $\mathrm{AK}$. As expected, the two deadline experiments, AHK and HKA, were significantly faster than the corresponding accuracy experiments. Comparison of the two deadline experiments showed that HKA was slower than AHK in all conditions, but the difference did not reach significance.

In evaluating the perceptual-interference effect, the irrelevant-noise condition was slower than the identical-noise condition in all experiments, with the difference ranging from 16 to $23 \mathrm{msec}$. It was significant in all comparisons; there were no significant interactions with experiments. The associative interference effect seen in a comparison of the irrelevantand incompatible-noise effects is also significant in all comparisons. The only significant interactions with experiments involved accuracy experi- ment HKA, in which the incompatible-noise condition was $32 \mathrm{msec}$ slower than that for irrelevant noise, compared with differences from 19 to $22 \mathrm{msec}$ in the other experiments. It is clear that these effects are robust and highly replicable.

Mean error rates are also included in Table 1. In the accuracy experiments, they are low. The deadline procedure was successful in inducing the speedaccuracy tradeoff in that these experiments are not only faster but produce considerably more errors than the corresponding accuracy experiments. In all instances, the highest error rates are in the incompatible-noise condition. In the deadline experiments, error rates for HKA are higher than for AHK in all conditions, but the difference reaches significance only for irrelevant noise $[\mathrm{t}(54)=2.99, \mathrm{p}<$ $.01]$. In spite of the lack of full support by statistical significance, the pattern of more errors in HKA, even with slower RTs, supports the conclusion that the HK discrimination is more difficult than the $\mathrm{AH}$.

The results presented here indicate that, with our equipment and procedures, we were able to confirm previous findings under conditions of both accurate and speeded performance. Not surprisingly, we have also shown that different letter combinations may produce significant differences in RTs in this situ-

Table 2

Values of $F(1,54)$ for Statistical Tests of Mean Reaction Time

\begin{tabular}{|c|c|c|c|c|c|c|}
\hline \multirow[b]{3}{*}{ Experiments Compared } & \multicolumn{6}{|c|}{ Source of Variance } \\
\hline & \multicolumn{3}{|c|}{$\begin{array}{l}\text { Identical and Irrelevant Noise } \\
\text { Conditions Compared }\end{array}$} & \multicolumn{3}{|c|}{$\begin{array}{l}\text { Irrelevant and Incompatible Noise } \\
\text { Conditions Compared }\end{array}$} \\
\hline & $\begin{array}{c}\text { Experi- } \\
\text { ment }\end{array}$ & $\begin{array}{c}\text { Noise } \\
\text { Condition }\end{array}$ & $\begin{array}{c}\text { Experiment } \\
\times \text { Noise } \\
\end{array}$ & $\begin{array}{c}\text { Experi- } \\
\text { ment }\end{array}$ & $\begin{array}{c}\text { Noise } \\
\text { Condition }\end{array}$ & $\begin{array}{l}\text { Experiment } \\
\times \text { Noise }\end{array}$ \\
\hline AHK Accuracy and AKH Accuracy & .56 & $72.70 \dagger$ & .79 & .66 & $64.62 \dagger$ & .18 \\
\hline AHK Accuracy and HKA Accuracy & $12.63 \dagger$ & $40.78 \dagger$ & .20 & $13.88 \dagger$ & $98.11 \dagger$ & $4.78^{*}$ \\
\hline AKH Accuracy and HKA Accuracy & $7.04 * *$ & $52.10^{\dagger}$ & 1.58 & $8.03^{* *}$ & $142.26 \dagger$ & $10.42^{* *}$ \\
\hline AHK Accuracy and AHK Deadline & $18.19 \dagger$ & $83.70 \dagger$ & .01 & $18.53 \dagger$ & $64.55 \dagger$ & .03 \\
\hline HKA Accuracy and HKA Deadline & $40.23 \dagger$ & $50.93 \dagger$ & .28 & $41.23 \dagger$ & $130.69 \dagger$ & $4.68^{*}$ \\
\hline AHK Deadline and HKA Deadline & 2.00 & $130.25 \dagger$ & .03 & 2.41 & $90.58+$ & .28 \\
\hline
\end{tabular}

*p<.05. $\quad * * p<.01 . \quad$ tp $<.001$. 
ation. Our main interest, however, is not in the mean RTs, but in information to be obtained by applying the measurement operations of variable-criterion theory to the RT distributions.

\section{THEORETICAL ANALYSIS}

According to the variable-criterion-theory conception, the excitatory strength (E) leading to response evocation grows as a function of time elapsed since stimulus onset, and when $E$ reaches the level of the response criterion $(\mathrm{C})$, the response occurs. Criterion level is regarded as the state of readiness to respond that exists at the beginning of the trial. The criterion is normally distributed over trials. The parameters of the criterion distribution are the mean $(\bar{C})$ and the standard deviation $(\sigma)$. The deterministic function for the growth of $\mathrm{E}$ and the normal distribution of criterion level generate the cumulative RT distribution. Theoretical functions for the growth of $\mathrm{E}$ may be estimated from empirical cumulative distributions in Thurstonean scale units. A short review of variable-criterion theory is presented in the Appendix. Included are the main scaling procedure, the response evocation characteristic (REC), and the race model applicable to CRT. Also presented are some additional details of the present analysis.

The logic of this analysis is to determine the functions for the growth of E separately for each noise condition. The temporal dynamics of perceptual and associative interference are then inferred from comparisons of these functions. Models for errors and for correct responses are included.

\section{Model for the AHK and AKH Experiments}

The first analysis of correct responding was of the AHK and AKH accuracy experiments because there were no significant differences between them. Functions for the growth of $\mathbf{E}$ were obtained from the cumulative group distributions at 20 -msec intervals, for each noise condition separately, for each of the two experiments. The patterns for the two experiments were essentially identical. Therefore, the functions for each noise condition for the two experiments were averaged after application of a scaling solution that corrected for trivial differences in the criterion parameters. The scale used was that of the AHK experiment. The values of $\bar{C}$ and $\sigma$ were 0 and 1. On this scale, the corresponding values for the AKH experiment were .086 and 1.027 .

Graphs of the fitted functions that very accurately describe the obtained ones for each noise condition are presented in the upper left panel of Figure 1. These functions provide an interesting and informative pattern. The functions for identical and irrelevant noise are negatively accelerated and have identical forms. However, the function for irrelevant noise is displaced below that for identical noise at a constant distance of .204 scale units. Thus, the effect of perceptual interference (PI) is a constant. This means that the effect is complete before the earliest responses in these experiments and does not affect the shape or the rate of growth of the function for E. ${ }^{2}$ As a model for these conditions, we suggest that the growth of $E$ for identical noise depends simply on the growth of associative strength and that $E$ for irrelevant noise is the same, although reduced by the constant for early perceptual interference. Thus, the first two expressions of the algebraic model are as follows:

Identical noise:

$$
E_{c}(t)=[A(t)-\bar{C}] / \sigma
$$

Irrelevant noise:

$$
E_{c}(t)=[A(t)-P I-\bar{C}] / o .
$$

$E_{c}(t)$ is the value of excitatory strength for the correct response at time $t$, following stimulus onset, and $A(t)$ is the associative strength at time $t . P I=.204$. $\bar{C}$ and $\sigma$ are the criterion parameters; for experiment $A H K$, they are 0 and 1 . The value of $A(t)$ in Equations 1 and 2 is given by the following exponential growth function:

$$
A(t)=2.676-17.737 e^{-.00461 t},
$$

with $\mathrm{t}$ expressed in milliseconds.

The function for incompatible noise was of a different shape. At the earliest latencies, it was superimposed with that for irrelevant noise, indicating an equivalent effect of perceptual interference. However, beyond $300 \mathrm{msec}$, it began gradually to fall below the irrelevant-noise function; it reached maximum separation at about $450 \mathrm{msec}$. Beyond this, the separation gradually decreased, and the functions were again superimposed beyond about $700 \mathrm{msec}$. Thus, the effect of associative interference begins later than that of perceptual interference and is a more dynamic process in the period during which responses occur. Completely absent at short latencies, it then waxes and wanes with the passage of time. For incompatible noise, associative strength is reduced both by the constant for perceptual interference and by a function describing the growth and decline of associative interference (AI). The expression for this is as follows:

$$
E_{c}(t)=[A(t)-P I-A I(t)-\bar{C}] / o,
$$

where $\mathrm{AI}(\mathrm{t})$ is the amount of associative interference at time $\mathrm{t}$.

The nonmonotonic course of $\mathrm{AI}(\mathrm{t})$ is described 

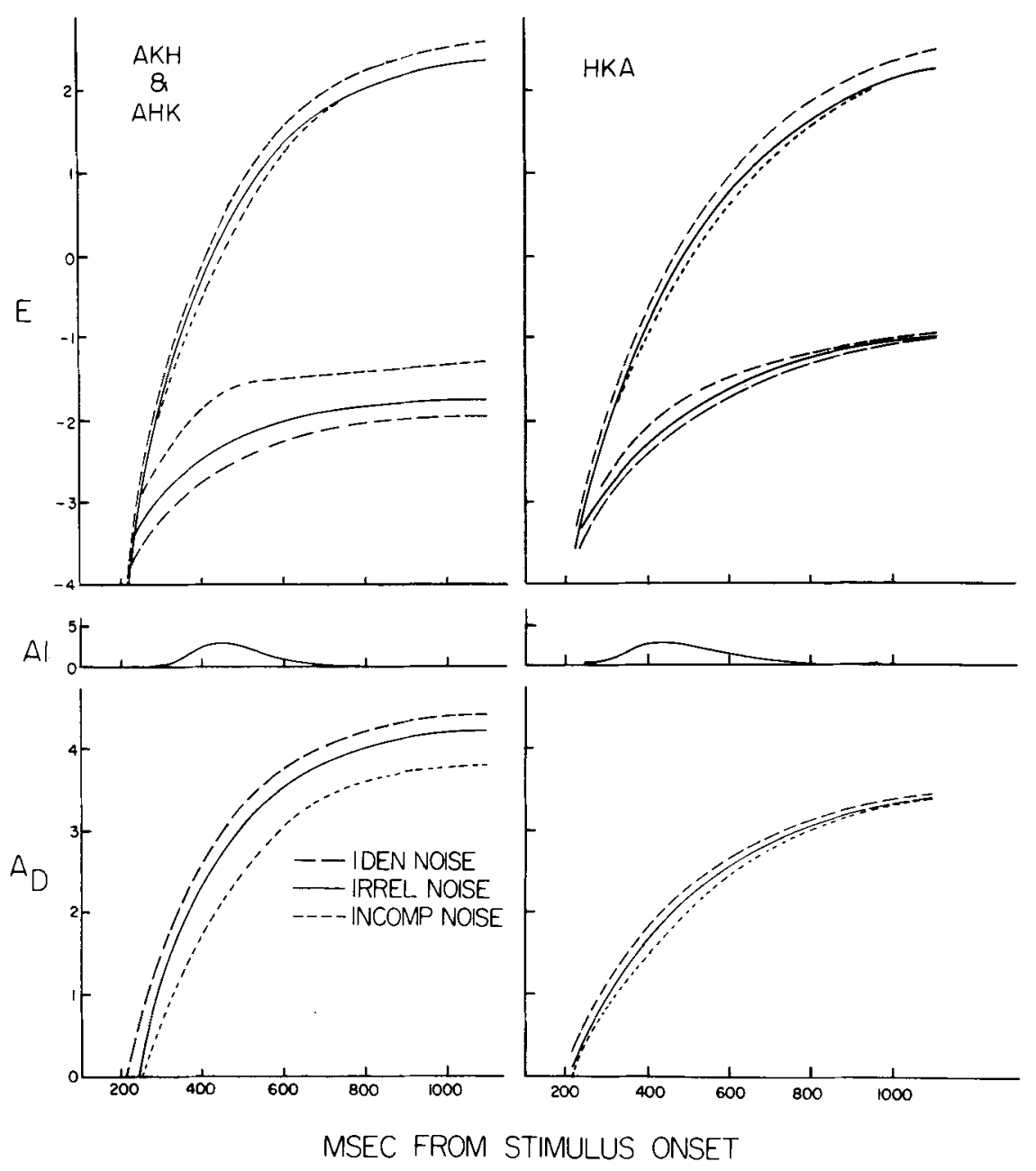

Figure 1. Graphic representation of the theoretical functions for the AHK and AKH experiments on the left and the HKA experiments on the right. The functions are for exclitatory strength (E), associative Interference (AI), and associative discrimination $\left(A_{D}\right)$. The three upper functions for $\mathbf{E}$ are for correct responses, and the three lower functions are for errors.

by a differential equation based on an inflected Gompertz growth function (Lewis, 1960, pp. 80-88; 182-185). The Gompertz function is of the form

$$
\mathrm{y}=\mathrm{vg}^{\mathrm{h}^{\mathrm{t}}} \text {, }
$$

where $\mathrm{v}$ is the asymptote, $\mathrm{g}$ is the intercept, and $\mathrm{h}$ determines the rate of growth. Our equation for AI is then of the form:

$$
A I(t)=d y=\frac{d y}{d t} d t
$$

The expression for calculating this function at 20 -msec intervals is

$$
A(t)=v g^{h^{t}} h^{t}(\ln g)(\ln h) \times 20 .
$$

For the fitted equation, the values of the parameters were $\mathrm{v}=3.446, \mathrm{~h}=.9877$, and $\mathrm{g}=1.202 \times 10^{-114}$. The very small value of the intercept means that the function was essentially asymptotic to zero until about $300 \mathrm{msec}$. The use of the differential equation may be taken to suggest that there is an underlying component of error strength that grows according to the Gompertz function and that the interference effect at any time is proportional to the rate of growth at that time. A graph of $\mathrm{AI}(\mathrm{t})$ is included in Figure 1.

The model for errors is necessarily based on the AHK deadline experiment. The functions for the growth of $E$ for correct responses and for errors in the deadline experiment were first obtained by applying the race model of Grice, Spiker, and Nullmeyer (1979). These data were then mapped into the accuracy model by RECs relating the correct $\mathrm{E}$ 
functions to the calculated values of Equations 1, 2, and 4. The fits were excellent, with an $r^{2}$ of .998 for two of the relations and .999 for the third. On the accuracy scale, the deadline values of 0 for identical, irrelevant, and incompatible noise were $1.083,1.041$, and 1.027. The corresponding values of $\bar{C}$ were $-.578,-.513$, and -.447 . As expected, the criterion levels are substantially lower for the deadline experiment. Since sampling error between conditions was somewhat larger than in the accuracy experiments, the separate estimates were used for added precision in the error analysis. The analysis was conducted on the original accuracy scale, so the six $\mathrm{E}$ functions were transformed to that scale.

As in the earlier theory of auditory CRT (e.g., Grice et al., 1982), we assume that associative discrimination $\left(A_{D}\right)$ increases with time, and that the function for the growth of error excitatory strength is as follows:

$$
E_{e}(t)=\left[A(t)-A_{D}(t)-\bar{C}\right] / \sigma .
$$

$E_{e}(t)$ is excitatory strength for errors at time $t$, and $A_{D}(t)$ is the amount of associative discrimination at $t$. Within this analysis, $\sigma=1$ and $\bar{C}=0$. The functions for the growth of $A_{D}$ were estimated by subtracting the obtained values of $E_{e}(t)$ from the calculated values of $A(t)$.

We next observed that the growth of $A_{D}$ in each condition was related in a rather precise linear fashion to the growth of correct excitatory strength for the condition. The values of $\mathrm{r}^{2}$ were .997 for identical and irrelevant noise and .999 for incompatible noise. The linear transformations by means of which $A_{D}$ may be calculated are as follows:

Identical noise:

$$
A_{D}(t)=.700 E_{c}(t)+2.697
$$

Irrelevant noise:

$$
A_{D}(t)=.725 E_{c}(t)+2.581
$$

Incompatible noise:

$$
A_{D}(t)=.731 E_{c}(t)+2.154
$$

The values of $E_{c}(t)$ are those from Equations 1, 2, and 4. A similar linear relation was also observed in the earlier auditory data, but has not previously been reported in the literature. It is of special interest here with respect to the incompatible-noise condition. It means the nonmonotonic function, $\mathrm{AI}$, which is a subtractive component of the correct function, is also present in the error function as an additive component. The relation also means that, except for the linear transformation, there are no terms in the error functions that are not also present in the correct functions.

There is one possible exception to the last statement. We have previously found that subjects differ in discriminative ability or the ability to inhibit errors. For this reason we introduce a weighting factor, $\mathrm{W}_{\mathrm{D}}$, or discrimination weight, which is applied to $A_{D}$. This is equivalent to saying that individuals differ in the linear transformation of $E_{c}$. If $W_{D}$ is other than 1, then Equation 8 becomes

$$
E_{e}(t)=A(t)-W_{D} A_{D}(t)-\bar{C} / o .
$$

The functions for $E_{e}$ and $A_{D}(t)$ are included in Figure 1.

At this point it is desirable to determine how well the model is able to predict the obtained cumulative distributions for the three experiments separately. Thus, the predicted proportions have been calculated using the predictive equations for the race model (Grice, Spiker, \& Nullmeyer, 1979). The calculated and obtained values for the three experiments are presented in Figure 2. For the accuracy experiments, only the incompatible-noise error distributions are shown. Also, data points for errors are not plotted. Visually, they would appear directly on the curves. For the deadline experiment, all values of $W_{D}$ were one. For the AHK accuracy experiment, they were $.874, .932$, and 1.082 for identical, irrelevant, and incompatible noise. For AKH, they were $.963,1.015$, and 1.142. However, the weights are not very reliable values when based on very low error rates. As a measure of goodness of fit, we present here the proportion of variance accounted for within the six distributions of each experiment. For AHK accuracy, AKH accuracy, and AHK deadline, the proportions were $.9998, .9991$, and .9993 , respectively. In the same order, the standard errors of estimating the data points within the six distributions were .004 , .007 , and .006 .

\section{Model for the HKA Experiments}

It was originally anticipated that the same model would apply to all of the experiments. The $\mathrm{E}$ functions for the HKA experiments were obtained in the same manner as above, and the pattern of plotted results was essentially similar. When the functions were plotted in an REC against the above model, however, the result was a nonlinear relation. The growth of associative strength was more gradual for the HKA data, with slower initial growth and greater continued growth at later times. Actually, this is a serendipitous discovery of some consequence. The important implication is that the discrimination of the target stimuli involves associative processes that continue for a considerable period of time following 

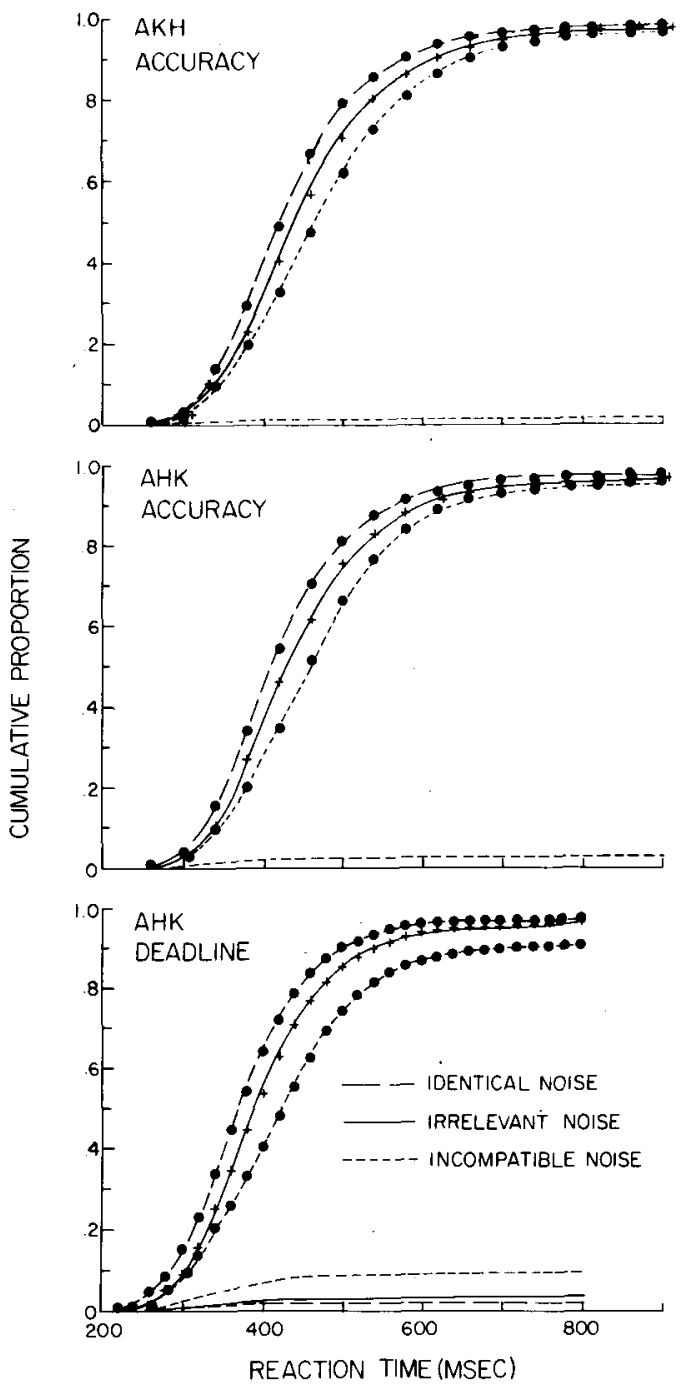

Figure 2. Calculated and obtained cumulative group distributions for each noise condition in the AHK and AKH experiments. Error distributions are included for only those conditions reaching at least $\mathbf{. 0 2}$. Data points not plotted for errors would appear directly on the curves.

stimulus onset. This evidence is also in full agreement with the continuous-flow conception of C. W. Eriksen and Schultz (1979).

As a way of evaluating the generality of the above model for perceptual and associative interference, we have derived, for the HKA experiments, a model with identical structure but different growth parameters. Since there was evidence for a little more sampling error in the criterion parameters between conditions than in the previous accuracy conditions, these have been estimated separately. The parameter estimates are shown in Table 3. It is seen that variation within experiments is small, and that criterion levels in the deadline experiment are again substantially below those of the accuracy experiment. Direct comparison of these values with those of the previous model is not possible, since nonlinear RECs do not allow estimates of the relations of criterion parameters.

As before, functions for the correct response were estimated from the accuracy experiment and functions for errors, from the deadline experiment. The exponential growth function for associative strength was as follows:

$$
A(t)=2.836-12.836 e^{-.00316 t} .
$$

The value of PI was .228. The nonmonotonic function for the growth of AI was of the same form but continued to be greater than 0 for a longer period of time. Parameter values for the Gompertz function were as follows: $\mathrm{V}=3.750, \mathrm{~h}=.9908$, and $\mathrm{g}=1.043 \times$ $10^{-63}$. Again, the growth of $A_{D}$ was linear with that of $\mathrm{E}_{\mathrm{c}}$. For identical, irrelevant, and incompatible noise, the values of $r^{2}$ were .998, .997, and .999. The linear transformations for calculating $A_{D}$ were as follows:

Identical noise:

$$
A_{D}(t)=.547 E_{c}(t)+1.944
$$

Irrelevant noise:

$$
A_{D}(t)=.576 E_{c}(t)+1.933
$$

Incompatible noise:

$$
A_{D}(t)=.584 E_{c}(t)+1.869
$$

The rate of growth of $A_{D}$ with $E_{c}$ is consistently smaller than with the previous experiments. For comparison, the model for the HKA experiments is presented graphically on the right side of Figure 1 . The more gradual growth of $\mathbf{A}$ is observable. The separations of the error functions and the $A_{D}$ functions are less than in the other model. Presumably, this is related to the greater confusability of the target letters.

Fits to the cumulative group distributions of the two HKA experiments have been made using the

Table 3

Criterion Parameters for Each Condition in the HXA

Experiments on the Scale of the Accuracy Irrelevant-Nolse Condition

\begin{tabular}{lccccc}
\hline \multirow{2}{*}{$\begin{array}{c}\text { Noise } \\
\text { Condition }\end{array}$} & \multicolumn{2}{c}{ Accuracy Experiment } & \multicolumn{2}{c}{ Deadline Experiment } \\
\cline { 5 - 6 } \cline { 5 - 6 } & $\overline{\mathrm{C}}$ & $\sigma$ & & $\overline{\mathrm{C}}$ & $\sigma$ \\
\hline Identical & .108 & 1.043 & -.827 & .875 \\
Irrelevant & .000 & 1.000 & -.827 & .875 \\
Incompatible & .085 & 1.000 & -.788 & .916 \\
\hline
\end{tabular}




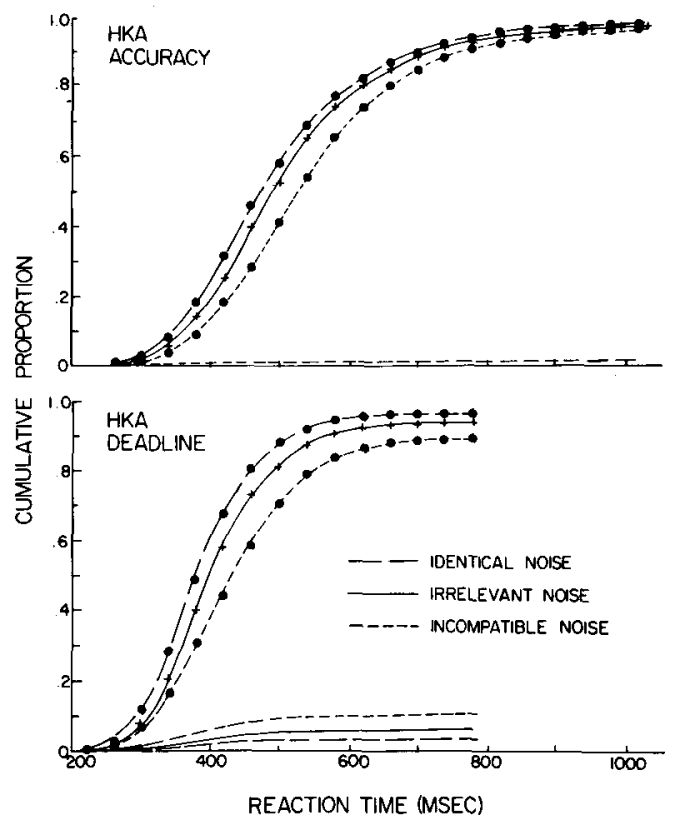

Figure 3. Calculated and obtained cumulative group distributons for each noise condition in the HKA experiments. Error distributions are included for only those conditions reaching at least .02. Data points not plotted for errors would appear directly on the curves.

present parameters, Equations 1, 2, 4, and 12 of the previous model, and the race model of Grice, Spiker, and Nullmeyer (1979). $W_{D}$ was 1 for the deadline experiment, and $1.187,1.166$, and 1.143 for the identical-, irrelevant-, and incompatible-noise conditions, respectively, in the accuracy experiment. The calculated distributions and the obtained proportions are shown in Figure 3. For both experiments, the proportion of variance accounted for within the six distributions was .9999 , and the standard error of estimate, also within distributions, was .003 . This rather extraordinary goodness of fit indicates something about not only the adequacy of the model, but the orderliness of the data as well.

\section{Application to Individual Subjects}

We regard the theories of information processing based on variable-criterion theory to be theories of the individual. However, they also apply to group data as long as the group criterion distribution approximates normality. Theoretical functions are estimated from group data or from within-subject data averaged over subjects, because of the much greater regularity of such data and because of the greater generality of such functions. ${ }^{3}$ Here, it is desirable to evaluate both the normality of the group criterion distribution and the applicability of the model to the individual subjects. Although this has been done repeatedly before, the present research does represent a shift of area. In order to do this, we have secured estimates, for each of the 140 subjects, of the criterion parameters in the three experimental conditions and fitted the six cumulative distributions for each subject. In order to do this, it was also necessary to estimate the individual-difference variable $W_{D}$ for each condition.

The general procedure is to obtain the $\mathrm{E}$ functions for each subject by applying the race model to each condition separately. Subject criterion parameters, on the scale of the model, are obtained from RECs relating the subject functions for the correct response to the appropriate calculated functions of the model. It is then possible to calculate the predicted distributions of correct responses and errors in the same manner as was done for the group distributions. The proportions were calculated at 40 -msec intervals. With 80 trials per condition, the results were surprisingly good for unpracticed subjects. The results were also uniform across the five experiments. The mean proportion of variance accounted for within the six distributions was .994 in each experiment. The median proportion was .995 in each experiment. The standard deviation of the goodness-of-fit measure across subjects averaged .003 for the five experiments. Only 8 of the 140 subjects had values of less than $\mathbf{9 9 0}$. It is clear that the model provides a satisfactory description of individual subject performance.

The fits of the subject distributions are a sufficient indication that the group distributions were a satisfactory source for the theoretical functions. However, we shall still evaluate the degree to which the group criterion distributions approximate normality. Grice et al. (1977) presented expressions that permit estimation of the first four moments of the group criterion distribution if the means and standard deviations of the subject distributions are known. Using the estimates of $\overline{\mathbf{C}}$ and $\sigma$ for each subject, these estimates have been made for each condition in each experiment. Based upon these estimates, the mean value of the index of skewness, $\gamma_{1}$, was -.068 , indicating essential symmetry. The mean value of the index of kurtosis, $\gamma_{2}$, was .112. This indicates leptokurtosis equal to that of a $t$ distribution of 57 degrees of freedom. The most extreme value of $\gamma_{2}$ was .4, which corresponded to a $t$ distribution of 19 degrees of freedom. Neither value would introduce significant distortion into scaling procedures based on normal assumptions.

In the case of experimental conditions or stimuli presented in an unpredictable order, response to all should be based on criteria from the same distribution (Grice, 1968). Thus, the three estimates of the criterion parameters here should differ only by sampling error. Of course, for individual subjects, the sampling error may be fairly sizable. We can present 
a descriptive measure that at least relates these within-subject differences to individual differences. We have computed the intraclass correlations between the three estimates of each of the parameters for the five experiments. For $\bar{C}$, the intraclass correlation varied from .92 to .98 , with a mean of .95 . For 0 it varied from .66 to .91 , with a mean of .79 . These values are about the same as those previously reported for SRT experiments with two stimulus intensities (Grice, Nullmeyer, \& Schnizlein, 1979; Grice, Spiker, \& Nullmeyer, 1982). The correlation is always smaller for o because there is much less individual difference variance in this variable. If, other than sampling error, there are additional unknown sources of within-subject variance in these variables, they are not major.

\section{Individual Differences Analysis of Error Rate}

In previous work of this kind, multiple regression analysis of the relation of error rate to the theoretical variables has been a useful procedure. Through the examination of individual differences, such an analysis may display some of the dynamics of information processing that are not made apparent in other ways. The usefulness of the method is limited to conditions producing an error rate sufficiently high to permit substantial individual difference variance. Here, the data analyzed are those of the incompatiblenoise conditions of the two deadline experiments. The multiple regression is for the prediction of error rate (ER) from the three individual difference variables: $\bar{C}, \sigma$, and $W_{D}$. One subject in the AHK deadline experiment was excluded because of failure to obtain a satisfactory estimate of $\mathrm{W}_{\mathrm{D}}$.

Intercorrelations of the four variables are presented in Table 4. The multiple correlation (R) was .950 in the AHK experiment and .986 in the HKA experiment. The multiple regression equations, in standard score form, were as follows:

AHK:

$$
\hat{\mathrm{z}}_{\mathrm{ER}}=-.946 \mathrm{z}_{\overline{\mathrm{C}}}+.454 \mathrm{z}_{o}-.933 \mathrm{z}_{\mathrm{W}_{\mathrm{D}}}
$$

HKA:

$$
\hat{z}_{\mathrm{ER}}=-.614 \mathrm{z}_{\overline{\mathrm{C}}}+.604 \mathrm{z}_{\sigma}-1.093 \mathrm{z}_{\mathrm{W}_{\mathrm{D}}}
$$

Further information concerning the analysis is presented in Table 5. The value of $F$ and the measure of importance or usefulness, $U$, are given for each variable. $U$ is the reduction in $R^{2}$ when the variable is deleted from the analysis.

Generally, the results confirm the findings of previous analyses of auditory CRT experiments. All variables contribute significantly to the regression, and the signs of all regression coefficients conform to
Table 4

Intercorrelations in the Incompatible-Nolse Condition of the Deadline Experiments of Error Rate (ER) and the Three Theoretical Variables: Criterion Mean $(\overline{\mathbf{C}})$, Criterion Standard Deviation (o), and Discrimination

\begin{tabular}{|c|c|c|c|c|c|c|}
\hline \multirow{2}{*}{$\begin{array}{l}\text { Vari- } \\
\text { able }\end{array}$} & \multicolumn{3}{|c|}{ AHK Deadline Experiment } & \multicolumn{3}{|c|}{ HKA Deadline Experimen } \\
\hline & $\overline{\mathrm{C}}$ & $\sigma$ & $W_{D}$ & $\overline{\mathrm{C}}$ & $\sigma$ & $W_{\mathbf{D}}$ \\
\hline $\begin{array}{l}\text { ER } \\
\bar{C} \\
\sigma\end{array}$ & -.510 & $\begin{array}{r}.478 \\
-.311\end{array}$ & $\begin{array}{r}-.210 \\
-.618 \\
.290\end{array}$ & .242 & $\begin{array}{l}.341 \\
.126\end{array}$ & $\begin{array}{r}-.749 \\
-.644 \\
.075\end{array}$ \\
\hline
\end{tabular}
Weight $\left(\mathbf{W}_{\mathbf{D}}\right)$

the predictions of the theory. One point that may seem initially puzzling is the significant negative regression weight of $\bar{C}$ in combination with a positive correlation with ER in the HKA experiment. This is not unusual. Actually, the correlation of -.51 between $\bar{C}$ and ER in the AKH experiment is an unusually high negative correlation, since the correlation is more often near 0 or positive. It appears that the level of $\bar{C}$ does affect error rate appropriately, but that individuals differ in discriminative ability as measured by $W_{D}$. Subjects relatively poor in discriminative ability tend to make errors and, as a result, may compensate by using higher, stricter criteria. The result will be a reduction in the potential negative correlation between $\bar{C}$ and ER, and the correlation may even become positive. For the HKA experiment, the partial correlation of $\bar{C}$ and ER, with $\mathrm{W}_{\mathrm{D}}$ held constant, is -.47 , and with $\sigma$ also held constant, the partial correlation is -.79 . For the AHK experiment, these values are -.83 and -.91 . Consistent with the interpretation is the finding that $W_{D}$ is a more important predictor of $E R$, relative to $\bar{C}$, in the HKA experiment. With the more difficult discrimination, individual differences in discriminative ability have more effect on ER, and with the easier discrimination, there is less constraint on criterion level. There is more individual difference variance of $\bar{C}$ in the AHK experiment. The standard deviations

Table 5

Values of F and Measures of Importance (U) for Regresaion Coefficients Predieting Error Rate in the IncompatibleNoise Condition of the Deadline Experiments From the Theoretical Variables: Criterion mean $(\bar{C})$, Criterion Standard Deviation (o), and Discrimination Weight $\left(W_{D}\right)$

\begin{tabular}{lccccc}
\hline & \multicolumn{2}{c}{$\begin{array}{c}\text { AHK Deadline } \\
\text { Experiment }\end{array}$} & & \multicolumn{2}{c}{$\begin{array}{c}\text { HKA Deadline } \\
\text { Experiment }\end{array}$} \\
${$\cline { 2 - 3 }$} }$ & $\mathrm{~F}(1,23)$ & $\mathrm{U}$ & & $\mathrm{F}(1,24)$ & $\mathrm{U}$ \\
\hline$\overline{\mathrm{C}}$ & 125.97 & .536 & & 116.83 & .138 \\
$\sigma$ & 42.89 & .183 & & 302.59 & .359 \\
$\mathrm{~W}_{\mathrm{D}}$ & 124.43 & .530 & & 369.17 & .437 \\
\hline
\end{tabular}

Note-For all values of $F, p<.001$. 
are .491 and .374 . The difference approaches significance $[F(26,27)=1.72, .10>p>.05]$.

As has been found in all previous analyses of this kind, the amount of criterion variability is related positively and significantly to ER. This is a prediction of the theory and, as an important factor in determining errors, has been missed by other approaches to the speed-accuracy tradeoff (Grice et al., 1982).

\section{DISCUSSION}

Our main goal in this research was, by examining their temporal dynamics, to get a better understanding of the processes of perceptual and associative interference produced by noise in the display. Our design, which used identical-, irrelevant-, and incompatible-noise stimuli, appears to have been successful in separating these processes. ${ }^{4}$ The effect of perceptual interference on latency has entirely ceased before responses occur-at least by about $250 \mathrm{msec}$. Beyond that time, it has no further effect, and associative strength grows at the same rate as in the identical-noise condition, but with no evidence of recovery from the initial deficit. With respect to the growth of associative discrimination, the effect has also ended before responding begins, but there is evidence for a small and gradual partial recovery from the deficit. The evidence for this is the slightly greater slopes of the functions relating $A_{D}$ to $E_{c}$ in the irrelevant-noise condition.

It comes as no great surprise that the effect of associative interference occurs later than that of perceptual interference, but at least we now have direct evidence that this is so. If a response occurs early, it may be correct or erroneous, but there is no effect upon latency. Beyond $300 \mathrm{msec}$, excitatory strength for the correct response in the incompatible-noise condition gradually falls behind the irrelevantnoise control as interference increases to its maximum. Beyond this time, there is gradual recovery, eventually from the entire deficit. It is of considerable interest that there is a secondary increase in error strength, an increase that is not present with irrelevant noise and that corresponds precisely with the increasing phase of interference. Following this, there is a period of decreasing gain in error rate, a decrease also not present with irrelevant noise. This corresponds precisely to the period of recovery for the correct response. Clearly, our analysis indicates that interference is related to the growth of error strength. But what kind of process could produce such a nonmonotonic effect?

At present, our best suggestion is that the interference effect involves an active inhibition of errors. At short latencies, inhibition is not effective and errors occur merely on the basis of their associative strength when the criterion for the response is low. With the passage of time, the ability to inhibit errors increases and some errors that would otherwise have reached criterion on the basis of associative strength are successfully inhibited. However, the inhibition may occur at some cost to the limited associative capacity available for the correct response. If the inhibition is successful, the delayed correct response will occur later, which would account for the phase of recovery from the interference effect. This interpretation is a possibility, but requires more direct evidence. It is certainly possible that the mere presence of a competing associative tendency directly weakens associative strength and produces a delay in response evocation. Another factor to be considered is that fixation is centered at the target letter. This implies that, on trials on which the fixation is well controlled, the noise letter will fall on a more peripheral portion of the retina and will take a little longer to become clear (C. W. Eriksen \& Schultz, 1977). This would account for the secondary rise of error strength and for the delay of the interference effect. It is possible that manipulation of the interstimulus distance would modify the temporal relationships. ${ }^{3}$

The effect of the discriminability of the target stimuli on the shape of the associative growth function was not the only possible outcome. It could have been an initial delay or possibly only an effect on the criterion. The evidence, however, indicates that discrimination of the target leading to the correct response is a continuously developing associative process that lasts for several hundred milliseconds. Grice, Spiker, and Nullmeyer (1979) obtained similar results with experiments in which tones differed in similarity. Another approach to the observation of discrimination is the examination of errors, in the present model indicated by the concept $A_{D} \cdot A_{D}$ also indicated slower growth in the HK experiments and thus replicated another finding of the earlier experiments with tones.

A potentially important finding here was the relation between the growth functions for correct responses and for errors in the incompatible-noise conditions and the presence of the same nonmonotonic process in both functions. Methodologically, it may be noted that this could not have been discovered without including the deadline experiments in the research. This is another example that supports the contention of Grice et al. (1982) that an adequate analysis of information processing in terms of RT requires attention to the dynamics of error production.

This research again confirms the principle that group RT distributions are of the same form and under the control of the same model as are the distributions of individual subjects. Since the work of Grice et al. (1977), this has been confirmed in at least 
17 published experiments, most of which contain two or more distributions. It appears that the time has come when an analysis based on group distributions no longer requires extensive justification. However, if an individual differences analysis is desired, the subject parameters must be obtained. In the case of disjunctive RT (c reaction), as opposed to simple and choice RT, more caution should be exercised in the use of group distributions, because of the possibility of individual differences in strategy (Grice et al., 1982).

\section{REFERENCES}

Eriksen, B. A., \& Eriksen, C. W. Effects of noise letters upon the identification of a target letter in a nonsearch task. Perception \& Psychophysics, 1974, 16, 143-149.

Eriksen, C. W., \& Schultz, D. W. Retinal locus and acuity in visual information processing. Bulletin of the Psychonomic Society, 1977, 9, 81-84.

Eriksen, C. W., \& Schultz, D. W. Information processing in visual search: A continuous flow conception and experimental results. Perception \& Psychophysics, 1979, 25, 249-263.

Grice, G. R. Stimulus intensity and response evocation. Psychological Review, 1968, 75, 359-373.

Grice, G. R. A threshold model for drive. In H. H. Kendler \& J. T. Spence (Eds.), Essays in neobehaviorism: A memorial volume to Kenneth $W$. Spence. New York: Appleton-CenturyCrofts, 1971.

Grice, G. R. Conditioning and a decision theory of response evocation. In G. H. Bower (Ed.), The psychology of learning and motivation (Vol. 5). New York: Academic Press, 1972.

GRICE, G. R. Accurate reaction time research with the TRS-80 microcomputer. Behavior Research Methods \& Instrumentation, $1981,13,674-676$.

Grice, G. R., Hunt, R. R., Kushner, B. A., \& Morrow, C. Stimulus intensity, catch trial effects, and the speed accuracy tradeoff in reaction time: A variable criterion theory interpretation. Memory \& Cognition, 1974, 2, 758-770.

Grice, G. R., Nullmeyer, R., \& Schnizlein, J. M. Variable criterion analysis of brightness effects in simple reaction time. Journal of Experimental Psychology: Human Perception and Performance, 1979, 5, 303-314.

Grice, G. R., Nullmeyer, R., \& Spiken, V. A. Application of variable criterion theory to choice reaction time. Perception \& Psychophysics, 1977, 22, $431-449$.

Grice, G. R., Nullmeyer, R., \& Spiker, V. A. Human reaction time: Toward a general theory. Journal of Experimental Psychology: General, 1982, 111, 135-153.

Grice, G. R., Spiker, V. A., \& Nullmeyer, R. Variable criterion analysis of individual differences and stimulus similarity in choice reaction time. Perception \& Psychophysics, 1979, 25, 353-370.

LEWIs, D. Quantitative methods in psychology. New York: McGraw-Hill, 1960.

Link, S. W. A comment on a new model for choice reaction time. Perception \& Psychophysics, 1979, 25, 443-446.

Thurstone, L. L. A method of scaling psychological and educational tests. Journal of Educational Psychology, 1925, 16, 433-451.

\section{NOTES}

1. C. W. Eriksen and Schultz (1979), using a three-letter display like that in the present experiments, obtained a mean RT of $434 \mathrm{msec}$ for a no-noise condition and a mean of $432 \mathrm{msec}$ for an identical-noise condition. The comparisons referred to here as "perceptual interference" involve differences of from 16 to $23 \mathrm{msec}$, with a mean of $19 \mathrm{msec}$ for the five experiments. All of the differences are statistically significant. The present research does not specifically address the question of whether or not any portion of these differences may be the result of facilitation by the same letter. The authors, however, have been unable to find clear evidence of facilitation by the same letter in experiments, similar to the present ones, with attention directed to a single fixed location and simultaneous presentation of the letters.

2. The functions for correct excitatory strength for identical and irrelevant noise in Figure 1 may appear to diverge in the vertical dimension, but do not. They do diverge in the horizontal dimension. The appearance of vertical divergence is a visual illusion that we have observed with other similar negatively accelerated functions.

3. As previously stated, the functions for the correct response were based on the group cumulative RT distributions. We actually began with an analysis of the AHK accuracy experiment based on the distributions of individual subjects as in most previous reports (e.g., Grice et al., 1982). In this procedure, the three functions were first obtained from the distributions of each subject. Then, all of the functions were converted to a common scale by a between-subjects scaling procedure. The means of all subject scale values were then computed at 20 -msec intervals for each noise condition. This produced a mean within-subject function for each condition. The trouble with this procedure for this research is that, at longer latencies, the means must be based on progressively fewer subjects. Scale values are not available for those subjects who have completed responding. The result is that the upper tails become unreliable and irregular. The problem is particularly relevant for the incompatible-noise condition, in which the relation is not a simple exponential growth function. This problem did not arise with the group functions.

We did, however, obtain smooth mean within-subject functions up to $660 \mathrm{msec}$, when $95 \%$ of responding was complete. Beyond that time, fewer than $50 \%$ of the subjects were contributing to the means. The availability of these functions makes possible a comparison with the model based on the group distributions. For each noise condition, the mean scale values were plotted against the calculated values from the algebraic model at $20-\mathrm{msec}$ intervals. The result was a precise linear relation for each noise condition. Moreover, the three linear relations were superimposed. A single linear regression applied simultaneously to the three relations yielded an $\mathrm{r}^{2}$ value of $\mathbf{9 9 9}$. Within this range, the two solutions lead to essentially identical conclusions about the temporal dynamics.

4. Reviewers have suggested that the effect of irrelevant noise may be a result, at least in part, of associative interference. The suggestion is that, based on featural similarity with a target, the irrelevant letter may produce some priming of the competing response. In view of related evidence obtained by B. A. Eriksen and C. W. Eriksen (1974), this is certainly a reasonable suggestion. Fortunately, this possibility can be evaluated for the present data because of the evidence for the greater similarity of $H$ and $K$ than of either of these letters to $\mathbf{A}$. This interpretation would predict a greater difference between identical and irrelevant noise when $H$ or $K$ was the irrelevant letter than when $A$ was the irrelevant letter. In the accuracy experiments, there is a very slight tendency in this direction, but the prediction is not supported by statistical significance. When the differences between identical and irrelevant noise are compared across experiments, with $\mathbf{A}$, rather than $\mathbf{H}$ or $\mathbf{K}$, as the irrelevant letter, there are no significant experiment $x$ noise type interactions. The differences are identical in the two deadline experiments.

There is another, somewhat more precise, prediction that may be evaluated. For example, in the AHK accuracy experiment, it is predicted that the irrelevant letter, $\mathbf{K}$, should produce a greater interference effect when $A$ is the target than when $H$ is the target. Because of $K$ 's greater similarity to $H$, there should be greater priming of the competing response in the case of the $A$ target. The 
data do not support this prediction. The negligible difference obtained is in the opposite direction. The mean difference between identical and irrelevant noise is $18.3 \mathrm{msec}$ when $A$ is the target and $19.8 \mathrm{msec}$.when $\mathrm{H}$ is the target. As indicated by the target $\times$ noise type interaction, the difference is not significant $[F(1,26)<1]$.

5 . It may be noted that the information about temporal dynamics provided by this kind of analysis is not the same as that which would be obtained by systematic manipulation of the stimulus onset asynchrony (SOA) of target and noise. Our information applies only to the specific condition-in this case, zero SOA. It could lead to hypotheses about other SOAs, but not to specific predictions. Application of the method to a number of SOAs should be a fruitful approach that provides complementary, but not redundant, information.

On the grounds that there must be variation in attention as well as in the criterion, one reviewer has objected to the use of variablecriterion theory in this situation. This merely reflects a lack of familiarity with the theory. From the outset, attention has been regarded as a determiner of criterion level and variation in attention as one of the factors contributing to criterion variability (e.g., Grice, 1972).

\section{APPENDIX}

Since the original proposal of variable-criterion theory by Grice (1968), there have been numerous applications in the areas of conditioning and RT research. The most recent summary of the theory was by Grice et al. (1982). With respect to response latency, the basic conception is that the stimulus information leading to response evocation grows as a monotonic, deterministic function of the time following stimulus onset. When this information reaches criterion strength, the response occurs. The criterion level is regarded as the readiness to respond or, alternatively, as the amount of information required for the decision to respond. Criterion level is multiply determined and is assumed to be normally distributed over trials with mean, $\bar{C}$, and standard deviation, $\sigma$. Criterion variability is treated as the sole source of variability, although a realistic conception is that $O$ is based on the sum of variances from all sources, among which criterion variability is large. Criterion level and its variability are thought of as being dependent on such factors as motivation, incentive, payoff conditions, emotional state, set, level of attention, adaptation, speed-accuracy instructions, and individual differences.

\section{Scaling Procedures}

Stimulus information leading to response evocation is called excitatory strength, E. Functions for the growth of $E$ are expressed as Thurstonean scale values in units of the standard deviation, $\sigma$, of the criterion distribution. The cumulative probability of response at any time is given by the integral of the unit normal density function from minus infinity to the value of $E$ at that time. Proportions on an empirical cumulative RT distribution provide estimates of the probability of reaching criterion by each time. Estimates of the scale values at each time are the normal deviates of the cumulative proportions. When a function for an entire distribution is estimated in this way, it is on a scale with $\sigma$ as the unit and $\bar{C}$ as the origin.

The most generally used scaling procedure is the response evocation characteristic, so named by Grice (1971) because of its close relation to the receiver operating characteristic (ROC) of signal detection theory. The model, however, is not the same as for the ROC, but is primarily based on the Thurstone (1925) model for the scaling of abilities. Consider two sets of data in scale values $x$ and $y$, which share common values of some variable, but may differ in the criterion parameters. In the present examples, the common variable is elapsed time since stimulus onset. If the scale values of set $y$ are plotted on the ordinate against the scale values of set $x$ on the abscissa at successive values of the common variable, the result will be linear if two conditions are satisfied: First, the criterion distributions are normal, and second, both data sets have the same relation to the common variable. The linear relation (Grice et al., 1977) is as follows:

$$
E_{y}=\frac{\sigma_{x}}{\sigma_{y}} E_{x}+\frac{\bar{C}_{x}-\bar{C}_{y}}{\sigma_{y}} .
$$

The slope estimates the ratio of the standard deviations, and the intercept estimates the difference between the criterion means. If we let $\sigma_{y}=1$ and $\bar{C}_{y}=0$, the relation becomes

$$
\mathrm{E}_{\mathbf{y}}=\sigma_{\mathbf{x}} \mathrm{E}_{\mathbf{x}}+\overline{\mathrm{C}}_{\mathbf{x}} \text {. }
$$

In other words, the slope now estimates $\sigma_{\mathrm{x}}$, and the intercept estimates $C_{x}$ on the $y$ scale. Equation 20 may be used for transforming the entire $x$ function to the $y$ scale. The reverse transformation may be made using the inverse parameters of Equation 20. One useful feature of such transformations is that when two or more functions are on the same scale, they may be averaged.

These parameter estimates are made by linear regression solutions. If both data sets are equally subject to error, then the appropriate solution is mutual regression, which minimizes the squared perpendicular residuals (Grice, Hunt, Kushner, \& Morrow, 1974). If only one data set is subject to error, or both are subject to error but one much more so than the other, then conventional unidirectional regression is appropriate. The less reliable data set is the predicted variable. An example of this would be the estimation of subject parameters in relation to a computed function.

One interesting example of REC analysis arose in the comparison of the AHK and AKH accuracy experiments. There were three noise conditions in each experiment, each with a different function for the growth of $\mathrm{E}$. Thus, there were three RECs relating the two experiments. Since the noise conditions were presented in an unpredictable order, the criterion parameters for each of the noise conditions should be the same except for within-subject sampling error. Each of the three RECs should estimate the same criterion parameters. In fact, when the three RECs were plotted together, they were almost precisely superimposed, indicating very small within-subject sampling error in these experiments. What was done was to treat the three linear relations as a single "hybrid"' REC and use a single mutual regression solution. The value of $r^{2}$ for this relation was .9976.

RECs that do not result in linear relations may also provide important information. This was the case when it was found that the growth of associative strength for the HKA experiments was not the same as for the other two letter combinations. 


\section{The Race Model}

The scaling model described above applies directly to experiments with a single response, such as simple RT and disjunctive RT with one response. In these experiments, proportions from the cumulative distribution direction estimate the theoretical probability that the response has reached criterion. This is not true for CRT with errors. In CRT, the trial is terminated by the first response, whether that response is correct or erroneous. Thus, on error trials, no information is provided as to the later time at which the correct response might have reached criterion. For this reason, empirical distributions of correct responses and errors do not separately provide estimates of the theoretical probabilities of reaching criterion. However, Grice, Spiker, and Nullmeyer (1979) presented the derivation of a race model that obtains these estimates for both correct responses and errors by utilizing both distributions in combination. They showed their equations to be a finite interval form of a model presented by Link (1979) for continuous functions. The finite interval form is required for the necessary computations.

When a model with functions providing calculated values of the theoretical probabilities is available, the following two equations provide the predicted RT distributions:

Correct responses:

$$
\Delta \mathrm{P}_{\mathrm{c}}(\mathrm{t})=\Delta \mathrm{F}_{\mathrm{c}}(\mathrm{t})\left[1-\mathrm{F}_{\mathrm{e}}(\mathrm{t})\right]-.5 \Delta \mathrm{F}_{\mathrm{c}}(\mathrm{t}) \Delta \mathrm{F}_{\mathrm{e}}(\mathrm{t})
$$

Errors:

$$
\Delta \mathrm{P}_{\mathrm{e}}(\mathrm{t})=\Delta \mathrm{F}_{\mathrm{e}}(\mathrm{t})\left[1-\mathrm{F}_{\mathrm{c}}(\mathrm{t})\right]-.5 \Delta \mathrm{F}_{\mathrm{c}}(\mathrm{t}) \Delta \mathrm{F}_{\mathrm{e}}(\mathrm{t}) .
$$

$\Delta \mathrm{P}_{\mathrm{c}}(\mathrm{t})$ and $\Delta \mathrm{P}_{\mathrm{e}}(\mathrm{t})$ are, respectively, the predicted proportions of correct responses and errors in a brief finite interval beginning at time t. $\Delta \mathrm{F}_{\mathrm{c}}(\mathrm{t})$ and $\Delta \mathrm{F}_{\mathrm{e}}(\mathrm{t})$ are the theoretical probabilities of each response's reaching criterion within the interval. $F_{c}(t)$ and $F_{e}(t)$ are the theoretical cumulative probabilities that the two responses have reached criterion before $t$. Predicted cumulative distributions are obtained by summating $\Delta \mathrm{P}_{\mathrm{c}}$ and $\Delta \mathrm{P}_{\mathrm{e}}$ over all $\Delta$ ts. These equations were used here for all predicted distributions, both for groups and individual subjects.

The equations for estimating the theoretical probabilities from empirical distributions are as follows:

For correct responses:

$$
\Delta \mathrm{F}_{\mathrm{c}}(\mathrm{t})=\frac{\Delta \mathrm{P}_{\mathrm{c}}(\mathrm{t})}{1-\mathrm{F}_{\mathrm{e}}(\mathrm{t})}+\frac{.5\left[\Delta \mathrm{P}_{\mathrm{c}}(\mathrm{t}) / 1-\mathrm{F}_{\mathrm{e}}(\mathrm{t})\right]\left[\Delta \mathrm{P}_{\mathrm{e}}(\mathrm{t}) / 1-\mathrm{F}_{\mathrm{c}}(\mathrm{t})\right]}{1-\mathrm{F}_{\mathrm{e}}(\mathrm{t})} .
$$

For errors:

$$
\Delta F_{e}(t)=\frac{\Delta P_{e}(t)}{1-F_{c}(t)}+\frac{.5\left[\Delta P_{c}(t) / 1-F_{e}(t)\right]\left[\Delta P_{e}(t) / 1-F_{c}(t)\right]}{1-F_{c}(t)} .
$$

In this instance, $\Delta F_{c}(t)$ and $\Delta F_{e}(t)$ are the estimated theoretical probabilities of reaching criterion within the interval. $F_{c}(t)$ and $F_{e}(t)$ are the estimated theoretical probabilities that the responses have reached criterion before the interval. $\Delta \mathrm{P}_{\mathrm{c}}$ and $\Delta \mathrm{P}_{\mathrm{e}}$ are the obtained proportions of response within the interval. In applying the equations, both are first computed for the earliest nonzero interval and then successively for each interval in the distributions. $F_{c}(t)$ and $F_{e}(t)$ are incremented after each computation. The accuracy of these equations, which do contain some approximation, was confirmed by Grice, Spiker, and Nullmeyer (1979) for simulated data with known parameters. The equations were used here in obtaining from the data all of the functions for the growth of $E$ and in obtaining estimates of the subject parameters. The scaling model applies directly to these estimated probabilities to provide functions in scale units. One important feature of the procedure is that the shape of functions for correct responses is invariant with respect to error rate. When the functions are obtained, the parameters of expressions describing them are obtained by conventional curve-fitting methods.

\section{Letter Identification and CRT to Tones}

There is one significant difference between the model obtained here for letter identification and previous ones for CRT to tones (e.g., Grice et al., 1982). In those investigations, there was evidence that the earliest responses were based only on detection information and that only later was associative strength effective. The evidence was the repeated finding of an early linear relation between the growth functions and those from simple RT. We did conduct simple RT experiments in this situation and found no such evidence. We concluded, therefore, that responding in letter identification depends on the growth of associative strength from the beginning. The simple exponential growth functions that describe the present data for letter identification do not fit the biphasic form of growth found in the auditory experiments.

(Manuscript received April 19, 1982; revision accepted for publication August 3, 1982.) 